\title{
Nonlinear optimal perturbations in plane Couette flow
}

\author{
Luca Brandt, Yohann Duguet and Robin Larsson \\ Linné FLOW Centre, KTH Mechanics, SE-10044 Stockholm, Sweden \\ luca@mech.kth.se
}

Subcritical transition to turbulence can occur in a variety of wall-bounded shear flows when the laminar base state is not subject to linear instability. In this case, perturbations to the base state with a finite but very small amplitude can be amplified by non-normal effects, up to a level where nonlinear interactions come into play. Since transition is often undesired, leading to a dramatic increase in wall drag, it is important to know which kind of (weak) perturbations are susceptible to trigger it if we wish to delay it. A vast amount of litterature has described the linear mechanisms responsible for disturbance amplification. Optimisation methods have yielded linear optimal disturbances [1, 2], the disturbances which exhibit the largest linear growth. Yet the focus has been on linear amplification rather than on actual transition, which requires full nonlinearity to be taken into account. In this study, we go back to the original question of which perturbation is most likely to trigger transition to turbulence. We are hence investigating nonlinear optimal disturbances, those with smallest initial energy which effectively lead to a highly disordered flow. In the recent years, progress in the understanding of subcritical transition was made using the concept of 'edge states', originating from dynamical systems theory. 'Edge state' refers to the flow regime reached asymptotically by critical trajectories at the exact onset of transition. It is an unstable flow state and cannot be observed in experiments, but its stable manifold determines the basin of attraction of the base state. Direct numerical simulation in minimal domains of plane Couette flow has shown that the flow at the onset of transition asymptotically approaches an unstable finite-amplitude steady state solution, characterised by wavy streaks and streamwise rolls [3]. Using a shooting method, approach to such steady states is the way to characterise whether a transitional trajectory in phase space is actually an 'edge' trajectory.

In our investigation, we also focus on plane Couette flow in a computational box of size $4 \pi h \times 2 h \times 2 \pi h$. The Reynolds number (based on the half-gap $h$ ) used for most of the computations presented here is 400 . The numerical simulations are performed with a pseudospectral code using $48 \times 33 \times 48$ grid points in 
a)

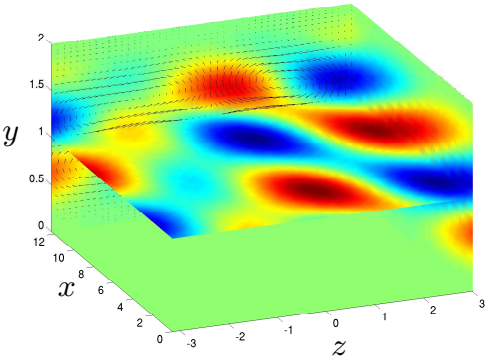

b)

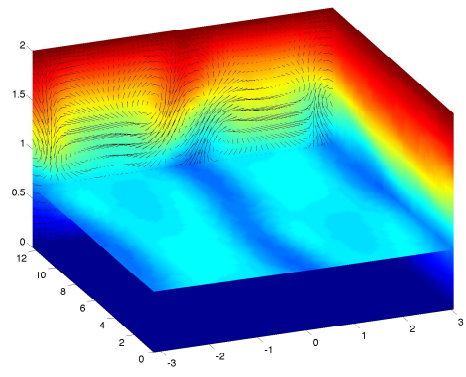

Fig. 1. a) Nonlinear optimal initial condition and b) corresponding symmetric edge state. The color indicates wall-normal velocity in $a$ ) and streamwise velocity in $b$ ). Velocity vectors represent in-plane velocities. The edge state has about 90 times more kinetic energy than the initial perturbation.

the streamwise, wall-normal and spanwise direction respectively. The method is though generalisable to all parallel shear flows. We use a finite-dimensional basis of linear optimal disturbances to generate candidates for the nonlinear optimal disturbances. Those linear optimal modes (and as well all linear combinaisons of them), satisfy two necessary conditions for nonlinear optimality: at time $t=0$, the first time derivative of the perturbation energy is zero while the second derivative is positive [4]. Only the linear optimal modes with lowest wavenumbers, i.e. those exhibiting the largest transient growth, are considered in the optimisation. We focus on two different transition scenarios involving departure from either oblique waves -with wavenumbers $(1, \pm 1)$ - or streamwise vortices with wavenumber $(0,2)$-, see also [5]. (The integers refers to multiples of the fundamental streamwise and spanwise wavenumbers, respectively)

For the range of parameters tested, the oblique wave scenario seems to be optimal, consistently with threshold studies in other shear flows [2]. The nonlinear interaction of two initial oblique waves of opposite angle and equal initial energy forces streamwise vortices. The lift-up effect then generates streaks and the whole structure approaches transiently the edge state. As a result of the optimisation, the nonlinear optimal initial condition is a symmetric pair of oblique waves, $(1, \pm 1)$, slightly perturbed by the mode $(1,2)$ with a phase departure of $0.6 \pi$. The distortion induced by the mode $(1,2)$ is small and corresponds to $9 \%$ of the energy of the total perturbation. The corresponding flow structure is shown in Fig. 1a) : the symmetric checkerboard patterns usually encountered in oblique transition is here distorted by the oblique mode $(1,2)$.

In the general case we find that not one, but three different steady state solutions can be transiently approached, depending on the exact shape and symmetries of the initial perturbations. Some of these steady states are new; one of them (denoted here 'Edge I') has lower energy than those previously found, 
a)

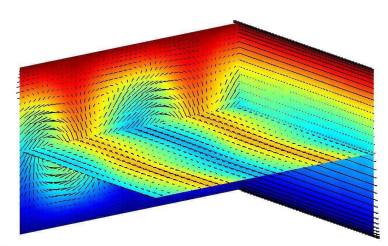

b)

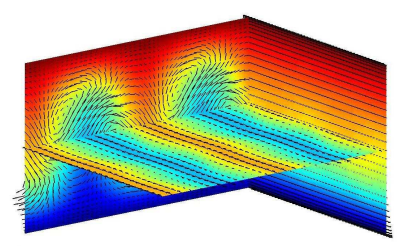

Fig. 2. $a$ ) Edge state II and $b$ ) edge state III. The color indicates streamwise velocity while velocity vectors represent in-plane velocities. Table 1 reports the energy of these steady solutions.

and displays low-speed streaks with a varicose structure, see Fig. 1b). It is the state transiently approached by optimal initial perturbations close to oblique waves. The two other edge states are approached transiently when perturbing linearly optimal streamwise vortices. They are displayed in Fig. 2. They are here refined down to machine accuracy using a Newton-Krylov solver. The steady solution denoted as edge state II has two wavy streamwise streaks with one sinuous oscillation within our computational box. It corresponds to the state observed in [3]. Edge state III, the most energetic among those found in our configuration, also has two streamwise streaks but with two streamwise oscillations.

The energy associated to the different edge states observed is reported in Table 1 . The minimal energy required by the simplest possible initial conditions approaching these is also compiled in the table. Edge II is reached by initial conditions consisting of streamwise rolls and a small amount of perturbation in the Fourier component $(1,2)$, of the order of few percents of the total energy. Conversely, the solution denoted by III is approached by a perturbation where the energy in the oblique mode $(1,1)$ is about half of that of the streamwise rolls.

\begin{tabular}{|c|c|c|c|}
\hline & Energy & Initial energy & Perturbation \\
\hline Edge I & $2.84 \times 10^{-3}$ & $3.34 \times 10^{-5}$ & $E_{(1,1)}=E_{(1,-1)}$ \\
Edge II & $1.82 \times 10^{-2}$ & $1.98 \times 10^{-4}$ & $E_{(1,2)}=0.0965 E_{(0,2)}$ \\
Edge III & $2.61 \times 10^{-2}$ & $1.72 \times 10^{-4}$ & $E_{(1,1)}=0.48 E_{(0,2)}$ \\
\hline
\end{tabular}

Table 1. Energy of the edge states identified with corresponding energy of the simplest initial conditions (only two Fourier modes) approaching them.

Finally, we investigate the Reynolds number behavior of the threshold energy associated to the oblique scenario and of the corresponding symmetric edge states. The energy thresholds are obtained by running the bisection algorithm using only the modes $(1, \pm 1)$ with equal energy while the steady solutions are computed by continuation starting from the solution at $R e=400$ 
in Fig. 1b). The perturbation energy associated to the symmetric edge state decreases asymptotically like $R e^{-0.5}$, whereas the initial energy required to approach these states decreases like $R e^{-1.9}$. The scaling of this optimal perturbation is very close to the optimal theoretical bound in $O\left(R e^{-2}\right)$ derived by Chapman [6].

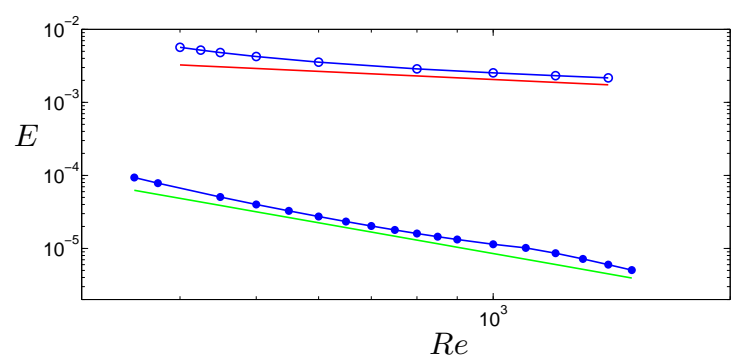

Fig. 3. Energy of the symmetric edge state I (open symbols) and of the symmetric initial condition with lowest energy lying on the laminar-turbulent boundary (filled symbol) versus the Reynolds number Re. The red and green lines represent best fitting power laws, $R e^{-0.5}$ and $R e^{-1.9}$ for the energy pertaining to the steady solutions and to the symmetric initial condition respectively.

The approach presented allows us to reconstruct the whole nonlinear transition process for critical optimal trajectories, starting from the linear regime. It also improves our understanding of the topology of the laminar-turbulent boundary in phase-space as $R e$ increases.

\section{References}

1. K. Butler and B. F. Farell, Three-dimensional optimal perturbations in viscous shear flow, Physics of Fluid, A 4, 1637 (1992).

2. P. J. Schmid and D. S. Henningson, Stability and transition in shear flows, Springer, 2001.

3. T. M. Schneider, J. F. Gibson, M. Lagha, F. De Lillo and B. Eckhardt, Laminarturbulent boundary in plane Couette flow, Phys Rev E, 037301 (2008).

4. C. Cossu, An optimality condition on the minimum energy threshold in subcritical instabilities, Comptes Rendus Mcanique, 333, 4, 331 (2005)

5. S. C. Reddy, P. J. Schmid, J. S. Baggett and D. S. Henningson, On the stability of streamwise streaks and transition thresholds in plane channel flows, Journal of Fluid Mechanics, 365, 269 (1998).

6. J.S. Chapman,Subcritical transition in channel flows, Journal of Fluid Mechanics, 451, 35 (2002) 